\title{
Estudio de caso único en gimnasia rítmica desde la perspectiva del coaching motivacional utilizando el modelo "La Jirafa de Cantón"
}

\section{Unique case study in rhythmic gymnastics from the perspective of motivational coaching using the model "Canton's Giraffe"}

Fecha de recepción: 04-02-2018

Fecha de aceptación: 22-04-2018

\begin{abstract}
Ana Isabel Sánchez Plazas
Psicóloga privada en ASPSICOLOGA .

Associació de Psicología de l'Esport de la Comunitat Valenciana (APECVA)

Violeta Expósito Boix

Línea de Motivación y Emoción Universidad de Valencia Associació de Psicología de l'Esport de la Comunitat Valenciana (APECVA)

David Peris-Delcampo

Universitat de Valencia: Departamento de Metodología y Máster en

Coaching y Liderazgo

Associació de Psicología de l'Esport de la Comunitat Valenciana (APECVA)

Enrique Cantón Chirivella

Universitat de València
\end{abstract}

\section{resumen/alostract:}

El actual trabajo presenta un diseño de caso único en una gimnasta rítmica de 11 años utilizando una metodología mixta de triangulación metodológica combinando un test-retest junto con el análisis cualitativo de las entrevistas realizadas. Para la intervención se utilizó el modelo de coaching motivacional "La Jirafa de Cantón" mediante un protocolo diseñado para su utilización. Se usaron los cuestionarios de Bienestar Psicológico de Ryff, la Escala de Autoestima de Rosenberg, la Escala de Autoeficacia General de Schwarzer y Bassler y la Escala de Autoeficacia para Niños y Adolescentes de Bandura.

Los resultados muestran una mejora de las puntuaciones de la escala específica para niños y adolescentes y un aumento de la apreciación subjetiva de los aspectos motivacionales y la percepción de recursos para lograr los objetivos planteados, tanto de la deportista como del entorno. Estos resultados están en la línea de otros estudios similares.

The present work presents a unique case design in an 11-year-old gymnast using a mixted methodology of methodological triangulation combining a test-retest along with the qualitative analysis of the interviews conducted. For the intervention, the motivational coaching model "La Jirafa de Cantón" was used through a protocol designed for its use, using The Psychological Wellbeing Scales of Ryff, the Rosenberg Self-esteem Scale, the General Self-Efficacy Scale of Schwarzer and Bassler and the Scale of Self-efficacy for Children and Adolescents of Bandura.

The results show an improvement of the scores of the specific scale for children and adolescents and an increase of the subjective appreciation of the motivational aspects and the perception of resources to achieve the objectives set, both of the athlete and the environment. These results are in line with other similar studies.

\section{palabras clave/keywords:}

intervención psicológica, coaching, motivación, gimnasia rítmica, Jirafa de Cantón spsychological intervention, coaching, motivation, rhythmic gymnastics, Canton's Giraffe 


\section{Introducción}

Cada vez es más importante encontrar y demostrar herramientas útiles para la intervención psicológica en diversos entornos y sobre todo en el deporte donde es cada vez más necesario optimizar el trabajo del profesional de la psicología (Cantón, 2016).

La reciente perspectiva de la psicología positiva (Seligman, 2008) ofrece un diferente punto de vista más orientado a la obtención de resultados y al desarrollo personal que a la eliminación del malestar o la enfermedad (Ortín, 2017). Dicha orientación está enfocada a analizar la salud mental de las personas y la calidad de sus vidas para responder a la pregunta: ¿qué podría llegar a ser esta persona? (Seligman y Czikszentmihalyi, 2000). Esta corriente de la psicología positiva se ajusta a las nuevas tendencias de intervención psicológica que busca esta optimización profesional.

Dentro de este enfoque positivo se engloba el coaching, que es un proceso mediante el cual un experto en esta metodología (llamado coloquialmente coach y en el caso de intervención psicológica a veces psicólogo-coach) genera un entorno facilitador donde el cliente o coachee "se da cuenta" y trabaja para lograr sus objetivos y avanzar en algún aspecto personal o profesional (Peris-Delcampo, 2014). Es una estrategia de modificación de conducta que, a través de la reflexión, el autodescubrimiento, el aprendizaje y la acción provoca un proceso de cambio (Garcia-Naveira, 2013) y que, sólo si la ocasión lo requiere, el experto en coaching (o coloquialmente coach) le aportará nuevas estrategias, diversas explicaciones y sugerencias (Buceta, 2016), a través de un proceso de acompañamiento individualizado donde el coachee, por medio de un plan concreto libera su talento (Villa y Caperán, 2010). Es necesario señalar, que en el coaching, el experto (coach) por un lado debe saber cómo generar ese proceso de cambio, y por otro ser competente respecto a qué se trabaja en ese proceso (Cantón, 2014b; Caperán y Peris-Delcampo, 2014; Peris-Delcampo, 2016, 2017).

Siguiendo esta idea y desde la base fundamentada y suficientemente probada demostración empírica de distintas teorías motivacionales clásicas como la Motivación de Logro (McClelland, 1989), el modelo de Autoeficacia (Bandura1997), la teoría de la Fuerza Motivacional o modelo de "Expectativas x Valor" (Atkinson, 1983; Vroom, 1964) y la Teoría de Metas (Deci y Ryan, 1985; Locke y Latham, 1990), Cantón (2013, 2014a) desarrolla un modelo de intervención motivacional desde la perspectiva de coaching bajo el nombre de "La Jirafa de Cantón" donde se trabajan diferentes componentes motivacionales con la figura de una jirafa siendo cuatro las partes a desarrollar en la intervención que a su vez representan cuatro grandes bloques de conceptos psicológicos relacionados con las teorías suficientemente probadas de motivación.

Intervenciones psicológicas desde el modelo "La Jirafa de Cantón" se han mostrado eficientes, tanto en natación adaptada (Peris-Delcampo, Expósito y Cantón, 2016) como en fútbol sala femenino (Colás, Expósito, Peris-Delcampo y Cantón, 2017), utilizando un protocolo de intervención (Cantón y Peris-Delcampo, 2017) específicamente diseñado para este modelo.

El presente trabajo muestra la intervención psicológica aplicando el modelo "La Jirafa de Cantón" en un diseño de caso único de una joven gimnasta rítmica utilizando una metodo- 
logía de triangulación metodológica (Castañer, Camerino y Anguera, 2013) por medio de la cual se utiliza un test-retest y un análisis cualitativo de las respuestas y las entrevistas realizadas. Se espera que haya una mejora en las puntuaciones de los cuestionarios, además de una percepción subjetiva de aumento de recursos eficaces para el logro de la meta planteada.

El objetivo de esta investigación fue incrementar la autoeficacia, el bienestar psicológico, la confianza y la motivación para mejorar el rendimiento a largo plazo de una gimnasta rítmica ayudándole en la consecución de su meta, mediante la técnica de "La Jirafa de Cantón" basada en un enfoque coaching.

\section{Descripción del caso}

La participante fue una gimnasta rítmica de 11 años que se encuentra en un proceso de duelo por la pérdida reciente de un progenitor. Cursa $6^{\circ}$ de Primaria y empezó a practicar este deporte desde los 9 años. Su meta es competir en campeonatos nacionales, por lo que entrena tres días a la semana, dos de ellos con todo el grupo de gimnasia rítmica y el otro día solo entrena con el grupo de competición del que forma parte. Ha tenido buen rendimiento y la entrenadora refiere que es una de las mejores dentro del grupo de competición.

Antes de comenzar la intervención se realizaron las acciones correspondientes a obtener el consentimiento informado tanto por parte de la gimnasta, como por parte de su tutor legal, aceptando las condiciones para la realización y presentación de este trabajo.

Se decide trabajar con ella mediante "La Jirafa de Cantón" por los siguientes motivos:

En los últimos entrenamientos ha tenido un decremento en el rendimiento deportivo, en consecuencia realiza ejercicios de menor dificultad.

En las competiciones comete más errores, por lo que prefiere realizar ejercicios más fáciles.

\section{Procedimiento}

En este estudio de caso único se ha utilizado un diseño mixto de triangulación metodológica combinando los métodos cuantitativos (test-retest con la administración de cuestionarios) con los cualitativos (entrevista semiestructurada siguiendo el guión de preguntas presentado en el Anexo 1) partiendo de un protocolo de intervención (Cantón y Peris-Delcampo, 2017) que se explicará más adelante.

\section{Evaluación}

Se ha aplicado el modelo motivacional "la Jirafa de Cantón" (Cantón 2013, 2014a) desde el cual se ha desarrollado la intervención. El modelo presenta cuatro partes diferenciadas que se van a describir brevemente:

La "Cabeza" que supone una metáfora de la meta, el logro u objetivo a alcanzar, definido adecuadamente en cuanto a su especificidad, realismo y una orientación adecuada.

El "Cuello" o las fuerzas que nos proyectan, que refleja metafóricamente las expectativas de eficacia y el valor de logro.

El "Cuerpo "o las tres bases de la confianza: la percepción de autoeficacia "puedo", la autoestima "valgo" y el autoconcepto "soy". 
Las cuatro "Patas" de la base que son una metáfora de la experiencia vicaria "lo que veo", la persuasión verbal "lo que oigo", los síntomas psicofisiológicos "lo que siento" y la experiencia directa "lo que hago".

Figura 1.- Modelo motivacional desde el enfoque coaching "La Jirafa de Cantón" (Cantón, 2013, 2014a).

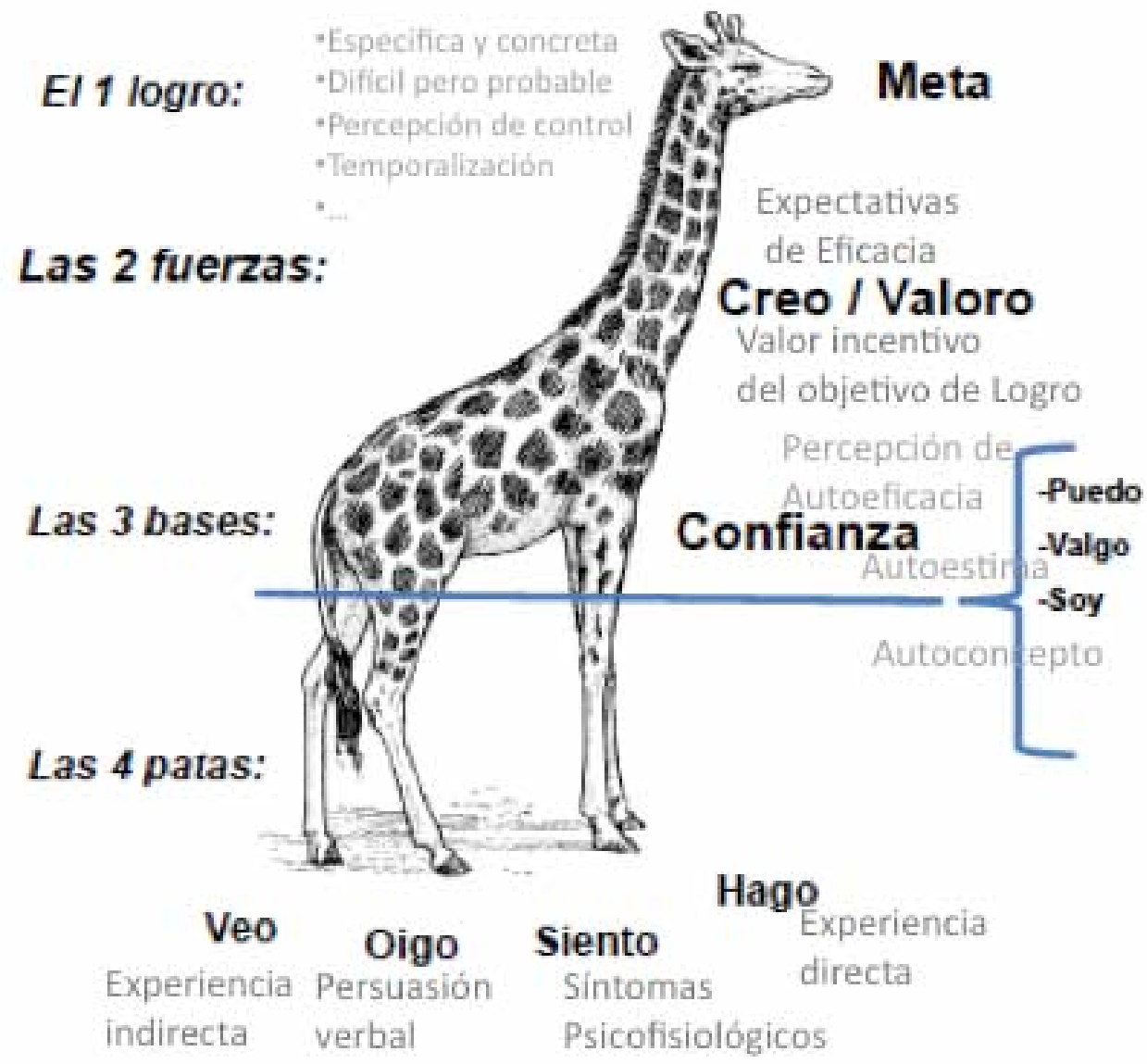

Para determinar el impacto de la estrategia motivacional basada en el coaching, "La Jirafa de Cantón" (Cantón, 2013) se han evaluado las variables psicológicas de autoestima, bienestar psicológico, autoeficacia general y autoeficacia en niños (al tratarse de una participante menor de edad) a través de los siguientes cuestionarios:

Escala de Autoestima de Rosenberg (1989) en su versión española de Vázquez-Morejón, Jiménez y Vázquez-Morejón (2004). Mide la autoestima entendida como sentimientos de valor personal y respeto hacia uno mismo con una alta fiabilidad (correlaciones en el test- 
retest entre $\alpha=.82$ hasta $\alpha=.88$ ). Esta prueba consta de 10 ítems en forma de pregunta, las cuales se puntúan en una escala de tipo Likert que varía entre 1 y 4 puntos, permitiendo obtener una puntuación máxima de 40 puntos y mínima de 10 puntos.

Escala de Bienestar Psicológico de Ryff (1989a, 1989b) en su versión española reducida de Díaz, Rodríguez-Cavajal, Blanco, Moreno-Jiménez, Gallardo,Valle y Van-Dierendonck, 2006). Esta escala está formada por 29 ítems medidos en una escala de seis puntos de tipo Likert; donde 1 es "completamente en desacuerdo" y 6 es "completamente de acuerdo" y se agrupan en seis dimensiones que presentaron una consistencia y análisis confirmatorio del modelo en esta escala de: Autoaceptación $(\alpha=.84)$ que mide la actitud de la persona hacia sí misma, Dominio del entorno $(\alpha=.82)$ que hace referencia a los entornos que crea la persona para facilitar el cumplimiento de sus objetivos, Relaciones positivas $(\alpha=.78)$ que se refiere a la capacidad de la persona para mantener relaciones sanas y basadas en la confianza, Propósito en la vida $(\alpha=.70)$ que mide la concreción de las metas de la persona, Crecimiento personal $(\alpha=.71)$ que es la capacidad de la persona para buscar situaciones que le permitan autorrealizarse personalmente, y Autonomía $(\alpha=.70)$ que hace referencia a si la persona es capaz de separar y mantener su identidad personal de la social.

Escala de Autoeficacia General (Schwarzer y Bassler, 1996), en su versión española de Sanjuán, Pérez-García y Bermúdez (2000). Evalúa el sentimiento estable de competencia personal para responder a situaciones potencialmente estresantes. Está formada por diez ítems sobre la percepción estable de competencia personal para manejar con éxito situaciones estresantes, los cuales se valoran en una escala de cuatro puntos de tipo Likert, siendo 1 "Nunca" y 4 "Siempre". La puntuación de la escala varía de 10 puntos hasta un total de 40 puntos como máximo. Presenta una fiabilidad entre 0.79 y 0.93 .

Escala de Autoeficacia para Niños y Adolescentes (Bandura, 1990) adaptada a la población española por Carrasco y Del Barrio (2002). Con una buena fiabilidad $(\alpha=.87)$, esta escala evalúa la percepción de eficacia de los niños (8-15 años) en distintos ámbitos de su vida como el académico, el social, el lúdico y el autocontrol. Consta de 35 ítems agrupados en tres dimensiones (autoeficacia académica, autoeficacia social y autorregulación) cuyo formato de respuesta está graduado de cero a cinco (1 "Fatal"; 2 "no muy bien"; 3"bien"; 4 y 5 "fenomenal").

\section{Intervención}

Antes de comenzar la intervención se tuvo contacto con la participante de la siguiente forma:

Presentación del profesional al grupo de competición donde participa la persona de manera dinámica (sentándose todos en círculo en el suelo). La acogida y la actitud de todas es buena.

Observación de los entrenamientos con el grupo de gimnasia de competición 5+1 (siempre hay una gimnasta de reserva que entrena con las demás).

Después, la intervención propiamente dicha se realizó en diez sesiones con una frecuencia semanal comenzando el 4 de Abril de 2017 y finalizando el 7 de Julio de 2017 y se dividió en tres fases, siguiendo el protocolo desarrollado por Cantón y Peris-Delcampo (2017): 
Fase de identificación (sesiones 1 a la 4) cuyos objetivos son recabar información mediante la administración de los cuestionarios e identificar los componentes de la motivación detectando fortalezas y debilidades mediante las entrevistas individuales donde también se trabajan aspectos psicoeducativos.

Fase de trabajo (sesiones 5 a la 9) donde se interviene sobre los diferentes aspectos motivacionales del modelo. Para esta tarea se emplean preguntas adecuadas a los componentes a trabajar en la sesión correspondiente. En todo momento el lenguaje y la forma de las preguntas son adecuadas a la edad de la participante.

Fase post-test (sesión 10 y seguimiento): es llevada a cabo al finalizar la intervención con el objetivo de evaluar su eficacia mediante la percepción subjetiva de la participante acerca de las sesiones y la administración de los cuestionarios anteriormente explicados.

Seguidamente, se presenta una tabla (Tabla 1) describiendo brevemente las sesiones que después se desarrollan más extensamente.

En la primera sesión se realiza una presentación y se crea un entorno adecuado de confianza para llevar a cabo la intervención. Después se realiza una entrevista semiestruturada en donde el objetivo es identificar las partes de la Jirafa de Cantón y explicar el modelo con un dibujo infantil de una jirafa, el cual tiene buena aceptación y le parece divertido. Antes de finalizar la sesión, se administran los cuestionarios anteriormente explicados.

La segunda sesión el objetivo se dedica a fomentar la participación y comprensión del modelo con el dibujo.

En la tercera y cuarta sesión el objetivo se trabaja en profundidad el modelo de la Jirafa y se identifican tanto los componentes de la motivación como las fortalezas y carencias.

La primera parte "la cabeza", en el modelo alude a las metas u objetivos. Al tratarse de una niña de 11 años se hace toda la evaluación con un dibujo de una jirafa que sirve para que sea más ameno explicar la metáfora de la Jirafa. Durante este proceso participa con entusiasmo y escribe en el dibujo en respuesta a las preguntas formuladas.

La meta que se plantea es "llegar muy lejos al campeonato de España". Este objetivo es claramente disfuncional por ser exigente, poco operativo y poco probable, no concreto y demasiado abstracto. En cuanto a las fortalezas, cuenta con apoyo familiar sobre todo de su madre y de sus abuelos que siempre la están animando, de su prima la cual ha sido entrenadora, y de amigos, en concreto de una de sus compañeras con la que ejecutaba los ejercicios en pareja más difíciles, le gusta la gimnasia rítmica y no supone un esfuerzo acudir a los entrenamientos a los que asiste siempre, por tanto, otra fortaleza será la buena adherencia a dichos entrenamientos, por lo que presenta una motivación adecuada para aplicar el modelo.

En la quinta sesión el objetivo es profundizar en las la identificación de fortalezas. Se trata de identificar las siguientes partes de la Jirafa: "cuello", "cuerpo" y "patas" con el dibujo.

El "cuello" del modelo basado en las expectativas de logro presenta ambivalencia, ya que la niña no está segura de que pueda conseguir las metas y que dependerá del día. El "creo" está correcto porque piensa que si se esfuerza puede alcanzar las metas. Por otro lado, el 
Tabla 1.- Descripción de las sesiones realizadas.

\begin{tabular}{|c|c|}
\hline Número de sesión & Objetivos de la sesión y trabajo realizado \\
\hline $\begin{array}{l}1(4 / 4 / 2017) \text { y } \\
2(11 / 4 / 2017) \\
\text { Identificación }\end{array}$ & $\begin{array}{l}\text { Entrevista semiestructurada con el objetivo de identificar las partes } \\
\text { del modelo "La Jirafa de Cantón". } \\
\text { Crear la confianza adecuada con la participante. } \\
\text { Explicación del modelo. } \\
\text { Detectar fortalezas y debilidades. } \\
\text { Aplicación pre-test de los cuestionarios. }\end{array}$ \\
\hline $\begin{array}{l}3(2 / 5 / 2017) \text { y } \\
4(16 / 5 / 2017) \\
\text { Identificación }\end{array}$ & $\begin{array}{l}\text { Identificación de la parte "cabeza" del modelo donde se observa } \\
\text { que el objetivo es poco operativo y es muy amplio. } \\
\text { Detección de fortalezas: cuenta con apoyo familiar, de su prima } \\
\text { que ha sido entrenadora y de sus amigos, además muestra una bue- } \\
\text { na adherencia a los entrenamientos. }\end{array}$ \\
\hline $\begin{array}{l}5(23 / 5 / 2017) \\
\text { Identificación }\end{array}$ & $\begin{array}{l}\text { Identificación de las restantes partes: "cuello"," cuerpo" y "patas" } \\
\text { del modelo, detectándose debilidades a trabajar en el "cuello", la } \\
\text { parte "soy" del cuerpo y en todas las "patas". } \\
\text { Fomentar la participación. }\end{array}$ \\
\hline $\begin{array}{l}6(6 / 6 / 2017) \\
\text { Trabajo }\end{array}$ & $\begin{array}{l}\text { Trabajo sobre la "cabeza" utilizando la técnica de la escalera "paso } \\
\text { a paso" para operativizar las metas. } \\
\text { Tareas propuestas: practicar técnicas de respiración, ejercicios de } \\
\text { flexibilidad y preguntar a la entrenadora cualquier aspecto que no } \\
\text { entienda. }\end{array}$ \\
\hline $\begin{array}{l}7(13 / 6 / 2017) \\
\text { Trabajo }\end{array}$ & $\begin{array}{l}\text { Revisión de tareas. } \\
\text { Fomentar la participación. } \\
\text { Trabajar la asertividad para hablar con la entrenadora. }\end{array}$ \\
\hline $\begin{array}{l}8(20 / 6 / 2017) \\
\text { Trabajo }\end{array}$ & $\begin{array}{l}\text { Revisión de tareas. } \\
\text { Fomentar la participación. } \\
\text { Trabajar la asertividad para hablar con la entrenadora. }\end{array}$ \\
\hline $\begin{array}{l}9(27 / 6 / 2017) \\
\text { Trabajo }\end{array}$ & $\begin{array}{l}\text { Revisión de tareas. } \\
\text { Fomentar la participación. } \\
\text { Trabajar la asertividad para hablar con la entrenadora. }\end{array}$ \\
\hline $\begin{array}{l}10(3 / 7 / 2017) \\
\text { Post-test }\end{array}$ & $\begin{array}{l}\text { Evaluación de la eficacia de la intervención. } \\
\text { Preguntas sobre sus opiniones acerca de la intervención y la per- } \\
\text { cepción de utilidad de las mismas. } \\
\text { Aplicación de los cuestionarios. }\end{array}$ \\
\hline
\end{tabular}


"valoro" es desproporcionado, ya que la niña piensa que ser campeona sería lo mejor para ella, piensa que su valor como persona depende de ello, se sentirán orgullosos los demás y ella misma, por lo que lo valora con un 10 sobre 10, lo que claramente es una expectativa muy poco realista. Por otra parte, estas expectativas se ven afectadas por la experiencia negativa de "tener nervios en las competiciones" y cree, que si no es "la mejor", la entrenadora no contará con ella, por lo que se detecta la necesidad de agradar y no defraudar a nadie. La expectativa de eficacia está afectada y no sabe si conseguirá sus objetivos.

El "cuerpo" del modelo está correcto puesto que basa la confianza en el esfuerzo, aunque el "soy" o autoconcepto está influenciado por la percepción de control en la situación, ya que no percibe control sobre el esfuerzo y lo condiciona a que no sabrá si estará nerviosa ese día. En cuanto al "valgo" tiene buena consideración de sí misma (se describe como ordenada, estudiosa y eso le ayuda a conseguir cosas). El "puedo" es adecuado porque quiere continuar esforzándose aunque se ve afectado por su falta de seguridad ante la competición, ya que lo condiciona a "ponerse nerviosa".

En cuanto a las cuatro patas del modelo se detecta que:

El "oigo" a nivel familiar está correcto porque su familia siempre la está apoyando y le refuerzan con mensajes positivos ("no te preocupes, lo haces bien"), aunque la entrenadora tiene un estilo castigador por lo que también escucha amenazas ("si no te sale te saco de la competición").

En el "veo" hay carencias porque no sabe cómo afrontar lo que le pasa. También se detecta una dualidad entre el apoyo de los familiares y amigas, y la crítica de su entrenadora. A la niña le gusta pensar en otras gimnastas que lo han conseguido y sostiene que es porque tienen más flexibilidad. A este respecto comentar que se ha establecido como una meta sencilla que busque ejercicios para trabajar en casa la flexibilidad y así pueda ver por sí misma sus progresos.

En el "hago" la niña acude tres veces por semana a entrenar y asiste a las competiciones. En verano entrena en un campamento de verano con otras gimnastas de su edad. Además, se detectan carencias, presenta conductas de evitación fisiológica, y conductas de seguridad. Al evitar conductas se encuentra mejor del nerviosismo por refuerzo positivo de la ansiedad, por lo que finalmente acaba no realizando actividades complejas y en las competiciones se suprimen los ejercicios de mayor dificultad efectuados sobre todo con aparatos (como la pelota o la cinta).

En el "siento", la niña cuenta que siente mucho nerviosismo y que está preocupada porque así no llegará a ser buena gimnasta. Las autoverbalizaciones son negativas: "no podré, seguro que me saca de la competición, la entrenadora no confía en mî́. Cabe señalar que tiene buenos entrenamientos y disfruta con ellos.

En la sexta sesión, el objetivo fue trabajar en profundidad "La Jirafa de Cantón" comenzando por las metas y se propusieron tareas. Primero, se operativizaron las metas para hacerlas más concretas y poder continuar. Para este propósito se utiliza la técnica de la escalera "paso a paso". Se hacen actividades con una escalera pintada en donde se van poniendo pequeñas metas más asequibles y funcionales. Al final del replanteamiento de metas, la 
deportista genera otra meta más concreta y que le hace ilusión: tener más flexibilidad como paso previo para estar mejor con su rendimiento y verse capaz de conseguir más metas poco a poco. Además y en relación con las carencias observadas se han propuesto las siguientes tareas: practicar una respiración que le enseñó hace tiempo su prima, practicar en casa ejercicios de flexibilidad preguntando a su prima cómo realizarlos de una manera más eficaz y darse cuenta de los pequeños logros que va consiguiendo, y preguntarle a la entrenadora sobre cualquier aspecto que no entienda durante los entrenamientos, ya que expresa que tiene miedo de preguntarle por "si le grita".

En la séptima, octava y novena sesión se han dedicado a revisar las tareas, reforzar la participación y proponer nuevas tareas.

En general las tareas han sido eficaces, la respiración le ha ido muy bien, se ha inspirado en una gimnasta de su pueblo para los ejercicios de flexibilidad, tiene menos miedo a hablar con la entrenadora y ha tenido conductas más asertivas porque se ha dirigido a la entrenadora para hacerle comentarios y después se sintió muy contenta porque le atendió en sus necesidades, por ejemplo, en un entrenamiento le preguntó directamente por el ejercicio y recibe feeback positivo.

Para percibir sus logros en flexibilidad, utilizó mensajes positivos que ella misma piensa ("si trabajo lo puedo alcanzar, lo hago bien, me esfuerzo más y me veo bien"). Además se inspiró en gimnastas de otro equipo próximo que compite en campeonatos de España.

Por último, la décima sesión se dedicó a realizar el pase de los cuestionarios para medir los resultados obtenidos. Después se preguntó a la participante sobre su opinión acerca de la intervención y sobre su percepción acerca del trabajo realizado. Al preguntarle si le había gustado el trabajo que había hecho en las sesiones de coaching a las que ella llamaba "La Jirafa", contesta que "me ha gustado mucho y me ha servido porque me siento mejor, más ilusionada y con ganas de entrenar y competir".

En una entrevista de seguimiento posterior en el mes de noviembre la entrenadora comenta la mejoría de la deportista.

\section{Resultados}

A continuación se presentan los resultados obtenidos en los cuestionarios administrados en la fase de identificación y en la fase de post-test.

Como se puede ver en la Tabla 2, las puntuaciones en autoestima no cambian después de la intervención, ya que se mantienen en 26. Sin embargo, hay tres ítems que aumentan su puntuación después de la intervención: el 4 ("Soy capaz de hacer las cosas como la mayoría de la gente") que pasa de 3 a 4 siendo importante este cambio dada su relación con la autoeficacia y la capacidad percibida, ya que esta niña se compara frecuentemente con las demás; el 6 ("Tengo una actitud positiva hacia mí mismo/a") que pasa de 1 a 2 mostrando un cambio de actitud en la participante puesto ha mejorado la visión de ella misma debido a la intervención y a los logros obtenidos en las tareas propuestas; y el 7 ("En general estoy satisfecho conmigo mismo/a") que pasa de 1 a 2, y, al igual que el anterior ítem, favorecería actitudes positivas hacia ella misma. 
Cabe señalar que, aunque el índice de autoeficacia también permanece estable con 37 puntos de un máximo de 40 puntos, hay determinados ítems que han variado su puntuación, concretamente el 5 ("Gracias a mis cualidades y recursos puedo superar situaciones imprevistas") pasa de 3 a 4 siendo esto importante dado que en su enunciado alude a la autoeficacia personal que influye directamente en el aumento de la motivación intrínseca, y el 7 ("Venga lo que venga, por lo general soy capaz de manejarlo") indica que su autoestima y la

Tabla 2.- Resumen pre y post-test en los cuestionarios administrados.

\begin{tabular}{|c|c|c|c|c|}
\hline Variable & Fase pre-test & & Fase post-test & Cambios \\
\hline \multirow[t]{2}{*}{ Autoestima } & \multicolumn{2}{|l|}{$26 / 40$} & $26 / 40$ & 0 \\
\hline & Autoaceptación & $21 / 24$ & $22 / 24$ & +1 \\
\hline \multirow{5}{*}{$\begin{array}{l}\text { Bienestar } \\
\text { Psicológico }\end{array}$} & $\begin{array}{l}\text { Relaciones } \\
\text { positivas }\end{array}$ & $29 / 30$ & $27 / 30$ & -2 \\
\hline & Autonomía & $11 / 36$ & $10 / 36$ & -1 \\
\hline & $\begin{array}{l}\text { Dominio } \\
\text { del entorno }\end{array}$ & $28 / 30$ & $30 / 30$ & +2 \\
\hline & $\begin{array}{l}\text { Crecimiento } \\
\text { personal }\end{array}$ & $23 / 24$ & $24 / 24$ & +1 \\
\hline & $\begin{array}{l}\text { Propósito } \\
\text { en la vida }\end{array}$ & $28 / 30$ & $28 / 30$ & 0 \\
\hline $\begin{array}{l}\text { Autoeficacia } \\
\text { General }\end{array}$ & & $37 / 40$ & $37 / 40$ & 0 \\
\hline \multirow{3}{*}{$\begin{array}{l}\text { Autoeficacia } \\
\text { en niños y ado- } \\
\text { lescentes }\end{array}$} & Académica & $56 / 68$ & $57 / 68$ & +1 \\
\hline & Social & $44 / 52$ & $45 / 52$ & +1 \\
\hline & $\begin{array}{l}\text { Regulación } \\
\text { emocional }\end{array}$ & $16 / 20$ & $17 / 20$ & +1 \\
\hline
\end{tabular}

percepción subjetiva de poder hacer algo a "priori" sin adelantarse con conductas de evitación o seguridad se han visto mejoradas.

Por otro lado, las principales variaciones tras la intervención se han concentrado especialmente en las subescalas de Autoeficacia en niños y adolescentes. La Autoeficacia académica percibida, la Autoeficacia social percibida y la Percepción de control aumentan un punto tras la intervención indicando una menor tendencia a la crítica y a la comparación 
social, además de una mayor cohesión del equipo de gimnasia rítmica y mejores relaciones sociales durante el tiempo de ocio (Autoeficacia social percibida). También, la participante ha adquirido una buena autorregulación que le permite una mejor consecución de sus metas y un adecuado manejo de sus emociones durante las competiciones (Percepción de control).

Además, se han obtenido variaciones en la subescala Dominio del entorno aumentando en dos puntos tras la intervención, lo cual es importante debido a la fase de duelo por la que está pasando. Esta circunstancia ha podido afectar a los resultados generales de la evaluación y de la medida post-test, pudiendo ser la causa las puntuaciones en Relaciones positivas con los otros y en Autonomía hayan sido más bajas y que, además, la subescala Propósito de vida no haya cambiado su puntuación.

\section{Discusión y conclusiones}

Tras analizar los resultados obtenidos en el test-retest y en el análisis cualitativo de las entrevistas realizadas, podemos concluir que la intervención psicológica mediante el enfoque coaching utilizando el modelo "La Jirafa de Cantón" ha mejorado la situación de la deportista, aumentando su percepción de que posee más recursos para hacer frente a sus próximos objetivos, aunque con ciertos matices que son necesarios analizar.

En primer lugar, la complicada situación de la gimnasta rítmica a nivel personal (tras haber perdido recientemente a un progenitor) marcó muy probablemente el proceso de la intervención, ya que como ella misma expresó, estaba reajustando su vida personal a esta nueva situación.

La deportista contestó a los cuestionarios de Bienestar Psicológico de Ryff (1989a, 1989b), Autoeficacia de Schwarzer y Bassler (1996) y el de Autoestima de Rosenberg (1989). Los resultados en estos cuestionarios no muestran cambios significativos respecto al retest en relación al pase inicial.

En cambio, al administrarle un cuestionario adaptado a la edad de la deportista (Escala de Autoeficacia para Niños y Adolescentes de Bandura, 1990), sí se mostraron diferencias dignas de reseñar: el retest mostró diferencias que nos hacen entender que la deportista mejoró en la aceptación de las críticas, la cohesión de grupo y relación social respecto a sus compañeras, una mayor percepción de control respecto a sus recursos para obtener sus metas y su percepción de dominar los aspectos relacionados con el entorno próximo. Como hemos comentado, su situación personal y estar actualmente en una fase de duelo marca su estado actual, lo que podría ser un factor que influya en la disminución de las puntuaciones de factores muy conectado con ello como son la valoración de las relaciones positivas con otros y autonomía, que son coherentes con su momento personal actual y las expresiones formuladas en las diferentes sesiones de trabajo.

Una conclusión plausible de la intervención es que podemos entender que se cumplen los objetivos planteados al principio de la misma, ya que han aumentado las puntuaciones del cuestionario específico para la edad de la deportista y la percepción subjetiva, tanto de la deportista como del entorno se muestra como positiva, entendiendo que percibe tener más recursos para lograr sus objetivos. Esto se demuestra en manifestaciones de la gimnasta 
del tipo de "me ha gustado mucho porque me siento mejor, más ilusionada y con ganas de entrenar y competir" o de la propia entrenadora que consideraba una mejoría notable de la deportista. Dichas conclusiones podrían haberse fortalecido con la valoración de conductas competitivas eficaces observables u otros indicadores objetivos de rendimiento (pactados con los entrenadores), siendo uno de los aspectos a mejorar en futuras investigaciones de este tipo.

Los resultados de este estudio están en la línea de otros anteriores donde se utilizó el procedimiento de "La Jirafa de Cantón", tanto en natación adaptada (Peris-Delcampo, Expósito y Cantón, 2016) como en fútbol sala femenino (Colás, Expósito, Peris-Delcampo y Cantón, 2017), en los que la intervención se mostró eficaz al mejorar la percepción motivacional de ambas deportistas respecto a sus objetivos de trabajo planteados.

Cabe destacar también, como limitación del trabajo, además de la anteriormente señalada, es la necesidad de adaptar este tipo de intervenciones a niños y adolescentes ya que, aunque el modelo "La Jirafa de Cantón" se entiende como útil para manejar los aspectos motivacionales de este tipo de deportistas, sí se considera adecuado buscar la manera de adaptar mejor la forma de realizar este tipo de intervenciones psicológicas en deportistas jóvenes, ajustándose a las necesidades de esta población.

El coaching es una muy buena herramienta psicológica que se muestra eficaz cuando se utiliza adecuadamente (Peris-Delcampo, 2014) y tiene una buena sustentación teórica con fundamentos psicológicos. Esto se demuestra en trabajos como el de Gil-Monte (2014), en el que desarrolla una fundamentación cognitivo-conductual del coaching, el empleo del "enfoque coaching" en el campo del liderazgo (Martínez-Moreno, 2014) o el diseño de un programa de intervención de coaching para deportistas jóvenes y su relación con el bienestar y salud psicológica (García-Naveira, García-Mas, Ruiz-Barquín, y Cantón, 2017). El modelo "La Jirafa de Cantón" sigue esta línea de trabajo buscando aplicar el coaching desde una fundamentación teórica y práctica desde la psicología, lo que augura buenas posibilidades de éxito en este tipo de intervenciones.

Es necesario este tipo de trabajos para que, cada vez más, las diferentes herramientas psicológicas aplicadas (en este caso el coaching) sirvan para los psicólogos "de campo" para realizar intervenciones óptimas, por lo que se recomienda realizar más estudios de este tipo que fundamenten estas intervenciones psicológicas.

\section{Referencias}

Atkinson, J.W. (1983). Personality, motivation and action. Nueva York: Praeger.

Bandura, A. (1990). Multidimensional scales of perceived self-efficacy. Stanford University, Stanford, C.A.

Bandura, A. (1997). Self-efficacy: The exercise of control. Nueva York, NJ: Freeman and Co. Publishers.

Buceta, J.M. (2016). Habilidades del Coach. Madrid: Universidad Nacional de Educación a Distancia.

Cantón, E. (2013). La "Jirafa de Cantón": un modelo de estrategias motivacional aplicado desde la perspectiva del Coaching. Granada: Editorial CSV. 
Cantón, E. (2014a). 'Cantón's Giraffe': A motivational strategy model applied from the perspective of coaching. The Coaching Psychologist, 10 (1), 26-34.

Cantón, E. (2014b). ¿El coaching es psicología? ¿Quién puede aplicar el Coaching y en qué entornos? Informació Psicologica, 107, 2-10. doi: http://dx.medra.org/10.14635/IPSIC.2014.107.1

Cantón, E. (2016). La especialidad profesional en Psicología del Deporte. Revista de Psicología Aplicada al Deporte $y$ al Ejercicio Físico, 1(e2), 1-7. doi: https://doi.org/10.5093/rpadef2016a2

Cantón, E. y Peris-Delcampo, D. (2017, julio). Psychological intervention in sport from the motivational coaching perspective. Comunicación presentada en el 14th World ISSP Congress of Sport Psychology. Sevilla, España. doi: 10.13140/RG.2.2.30882.73924

Carrasco Ortiz, M. A. y Del Barrio Gándara, M. V. (2002). Evaluación de la autoeficacia en niños y adolescentes. Psicothema, 14 (2), 323-332.

Castañer, M., Camerino, 0. y Anguera, M.T. (2013). Métodos mixtos en la investigación de las ciencias de la actividad física y el deporte. Apunts. Educación Física y Deportes, 112 (2), 31-55. doi: http://dx.doi.org/10.5672/ apunts.2014-0983.es(2013/2).112.01

Caperán, J.A. y Peris-Delcampo, D. (2014). Del coaching ejecutivo a otros campos de aplicación: una opción para optimizar el trabajo del psicólogo. Informació Psicologica, 107, 22-33. doi: http://dx.medra.org/10.14635/IP. SIC.2014.107.3

Colás, J.M., Expósito, V., Peris-Delcampo, D. y Cantón, E. (2017). Intervención psicológica desde el coaching motivacional utilizando el modelo "La Jirafa de Cantón" en una jugadora de fútbol sala. Revista de Psicología Aplicada al Deporte y al Ejercicio Físico, 2, e 10, 1-14. doi: https://doi.org/10.5093/rpadef2017a12

Deci, E.L. y Ryan, R.M. (1985). The general causality orientations scale: Self-determination in personality. Journal of Research in Personality, 19, 109-134. doi: https://doi.org/10.1016/0092-6566(85)90023-6

Díaz, D., Rodríguez-Carvajal, R., Blanco, A., Moreno-Jiménez, B., Gallardo, I., Valle, C. y Van-Dierendonck, D. (2006). Adaptación española de las escalas de bienestar psicológico de Ryff. Psicothema, 18 (3), 572-577.

García-Naveira, A. (2013). Aplicación profesional del coaching en el deporte: un estudio de caso único. Cuadernos de Psicología del Deporte, 13(2), 101-112. doi: https://doi.org/10.5944/ap.14.1.19255

García-Naveira, A., García-Mas, A., Ruiz-Barquín, R. y Cantón, E. (2017). Programa de intervención basada en el coaching en jóvenes deportistas de alto rendimiento, y su relación con la percepción de bienestar y salud psicológica. Revista de Psicología del Deporte, 26(2), 37-44.

Gil-Monte, P. (2014). Coaching cognitivo-conductual: una herramienta para los psicólogos. Informació Psicològica, 107, 34-46. doi: http://dx.medra.org/10.14635/IPSIC.2014.107.4

Locke, E. A., y Latham, G. P. (1990). A theory of goal setting and task performance. Englewoods Cliffs, NJ, Estados Unidos: Prentice-Hall.

Martínez-Moreno, A. (2014). Liderazgo y Coaching. Informació Psicologica, 107, 47-65. doi: http://dx.medra. org/10.14635/IPSIC.2014.107.5

McClelland, D. C. (1989). Estudio de la Motivación Humana. Madrid, España: Narcea.

Ortín, F. J. (2017). El coaching desde el enfoque positivo de la Psicología. Valencia, España: Certificado Universitario en Psicología Positiva y Coaching de ADEIT -Universidad de Valencia.

Peris-Delcampo, D. (2014). El coaching: cómo podemos utilizarlo con éxito. Informació Psicològica, 107, 11-22. https://doi.org/10.14635/IPSIC.2014.107.2

Peris-Delcampo, D. (2016). Nociones básicas de psicología del deporte y coaching para gestores de empresas deportivas. Almería, España: Círculo Rojo.

Peris-Delcampo, D., Expósito, V. E. y Cantón, E. (2016). Intervención psicológica en natación adaptada desde la perspectiva del coaching motivacional. Informació Psicològica, 112, 59-73. https://doi.org/10.14635/IPSIC.2016.112.5. 
Rosenberg, M. (1989). Society and the adolescent self-image. Middletown, CT, España: Wesleyan University Press.

Ryff, C. (1989a). Beyond Ponce de Leon and Life satisfaction: New directions in quest of successful aging. International Journal of Behavioral Development, 12, 35-55. doi: https://doi.org/10.1177/016502548901200102

Ryff, C. (1989b). Happiness is everything, or is it? Explorations on the meaning of psychological well-being. Journal of Personality and Social Psychology, 57, 1069-1081. doi: https://doi.org/10.1037/0022-3514.57.6.1069

Sanjuán, P., Pérez-García, A. M. y Bermúdez, J. (2000). Escala de autoeficacia general: datos psicométricos de la adaptación para la población española. Psicothema, 12, 509-513.

Seligman, M.E.P. (2008). Positive Health. Applied Psychology: An International Review, 57, 3-18. doi: 10.1111/j.14640597.2008.00351.x

Seligman, M.E.P. y Csikszentmihalyi, M. (2000). Positive Psychology: An Introduction. American Psychologist, 55 (1), 5-14. doi:10.1037/0003-066X.55.1.5

Schwarzer, R. y Baessler, J. (1996). Evaluación de la autoeficacia: adaptación española de la escala de Autoeficacia General. Ansiedad y Estrés, 2 (1), 1-8.

Vázquez-Morejón, A., Jiménez, R. y Vázquez-Morejón, R. (2004). Escala de Autoestima de Rosenberg: fiabilidad y validez en población clínica española. Apuntes de Psicología, 22 (2), 247-255.

Villa, J. P. y Caperán, J. A. (2010). Manual de Coaching. Barcelona, España: Profit Editorial.

Vroom, V. H. (1964), Work and Motivation. New York, NY, Estados Unidos: John Wiley and Sons.

Anexo 1. Preguntas utilizadas durante la entrevista semiestructurada EL LOGRO: LAS METAS (CABEZA)

\begin{tabular}{ll}
\hline Generales & ¿Cuál es tu objetivo ahora mismo? \\
& ¿Qué quieres conseguir exactamente? \\
\hline Concretas & ¿Crees que es específica o que se refiere a otras \\
& cosas de tu vida? \\
& ¿Crees que se puede dividir en partes más \\
& pequeñas para conseguir la meta en gimnasia \\
& rítmica? \\
\hline Percepción de probabilidad & ¿Cuándo quieres alcanzarla? \\
& ¿Qué crees que puedes necesitar? \\
\hline Percepción de control & ¿Te sientes apoyado para conseguir la meta? \\
& ¿Cómo harías los cambios para lograrla? \\
\hline Temporalización & ¿Cómo te vas a organizar? \\
& ¿Cómo piensas que se podrían comprobar las \\
& mejoras? \\
\hline
\end{tabular}




\section{LAS FUERZAS MOTIVACIONALES (CUELLO)}

\begin{tabular}{ll}
\hline Expectativas de autoeficacia: & ¿Crees que puedes conseguir la meta? \\
"Creo" & ¿Es importante para ti esta meta? \\
\hline Valor del incentivo: "Valoro" & De 0 a 10, ¿cuán de importante es para ti la meta? \\
& ¿Cómo valoras tú conseguir la meta? \\
\hline
\end{tabular}

\section{LA CONFIANZA: PUEDO/ VALGO/ SOY (CUERPO)}

\begin{tabular}{ll}
\hline Autoeficacia: "Puedo" & ¿Confías en ti misma para lograr la meta? \\
& ¿Piensas que lo conseguirás? \\
\hline Autoestima: "Valgo" & ¿Piensas que pones todo de tu parte para \\
& conseguirlo? \\
& ¿A qué crees que se debe que el resultado que \\
& consigas sea positivo o negativo? \\
\hline Autoconcepto: "Soy" & ¿Qué cualidades tienes para conseguirla? \\
& ¿Cómo te imaginas consiguiendo la meta? \\
\hline
\end{tabular}

\section{LAS FUERZAS MOTIVACIONALES (CUELLO)}

\begin{tabular}{ll}
\hline $\begin{array}{l}\text { Expectativas de autoeficacia: } \\
\text { "Creo" }\end{array}$ & $\begin{array}{l}\text { ¿Crees que puedes conseguir la meta? } \\
\text { ¿Es importante para ti esta meta? }\end{array}$ \\
\hline Valor del incentivo: "Valoro" & $\begin{array}{l}\text { De } 0 \text { a } 10, \text { ¿cuán de importante es para ti la meta? } \\
\text { ¿Cómo valoras tú conseguir la meta? }\end{array}$ \\
\hline LA CONFIANZA: PUEDO/ VALGO/ SOY (CUERPO) \\
\hline Autoeficacia: "Puedo" & ¿Confías en ti misma para lograr la meta? \\
& ¿Piensas que lo conseguirás? \\
\hline Autoestima: "Valgo" & ¿Piensas que pones todo de tu parte para \\
& conseguirlo? \\
& ¿A qué crees que se debe que el resultado que \\
& consigas sea positivo o negativo? \\
\hline Autoconcepto: "Soy" & ¿Qué cualidades tienes para conseguirla? \\
& ¿Cómo te imaginas consiguiendo la meta? \\
\hline
\end{tabular}




\section{LAS BASES: LA EXPERIENCIA (PATAS)}

\begin{tabular}{ll}
\hline $\begin{array}{l}\text { Experiencia indirecta: "Lo que } \\
\text { veo" }\end{array}$ & $\begin{array}{l}\text { ¿Te has fijado en alguien para plantearte esta } \\
\text { meta? }\end{array}$ \\
\hline ¿ersuasión verbal: "Lo que & ¿Qué opina tu familia? \\
oigo" & ¿Qué tipo de apoyo te dan los demás? \\
\hline Síntomas psicofisiológicos y & ¿Qué sientes cuando piensas en la meta? \\
emociones: "Lo que siento" & ¿Cómo te afectan los comentarios de la \\
& entrenadora y de los demás? \\
\hline Experiencia directa: "Lo que & ¿Te habías planteado alguna meta similar a esta? \\
hago" & ¿Qué resultados has obtenido en metas similares? \\
\hline
\end{tabular}

